\title{
Natural history of sensitization, symptoms and occupational diseases in apprentices exposed to laboratory animals
}

\author{
D. Gautrin*, H. Ghezzo*, C. Infante-Rivard", J-L. Malo*
}

\begin{abstract}
Natural history of sensitization, symptoms and occupational diseases in apprentices exposed to laboratory animals. D. Gautrin, H. Ghezzo, C. Infante-Rivard, J-L. Malo. (C) ERS Journals Ltd 2001.

ABSTRACT: The natural history of the development of sensitization and disease due to high-molecular-weight allergens is not well characterized. This study describes the timecourse of the incidence of work-related symptoms, skin reactivity and occupational rhinoconjunctivitis (RC) and asthma (OA); and assesses the predictive value of skin testing and $\mathrm{RC}$ symptoms in apprentices exposed to laboratory animals, in a 3-4-yr programme.
\end{abstract}

Four-hundred and seventeen apprentices at five institutions were assessed prospectively with questionnaire, skin-testing with animal-derived allergens, spirometry and airway responsiveness $(n=373)$. Depending on the school, students were seen 8 $(n=136), 20(n=345), 32(n=355)$ and $44(n=98)$ months after starting the programme.

At all visits, the incidence was greater for work-related RC symptoms followed in order by skin reactivity, occupational $\mathrm{RC}$, and, almost equally, $\mathrm{OA}$ and work-related respiratory symptoms. The incidence-density figures were comparable for each followup period and for most indices up to 32 months after entry into the study and then tended to decrease. The positive predictive values (PPVs) of skin reactivity to workrelated allergens for the development of work-related $\mathrm{RC}$ and respiratory symptoms were $30 \%$ and $9.0 \%$, respectively, while the PPVs of work-related RC for the development of $\mathrm{OA}$ was $11.4 \%$.

Sensitization, symptoms and diseases occur maximally in the first 2-3 yrs after starting exposure to laboratory animals. Skin reactivity to work-related allergens and rhinoconjuctivitis symptoms have low positive predictive values.

Eur Respir J 2001; 17: 904-908.
*Dept of Chest Medicine, Hôpital du Sacré-Coeur, Montreal, Canada and ${ }^{\#}$ Joint Depts of Epidemiology and Biostatistics and Occupational Health, McGill University, Montreal, Canada.

Correspondence: D. Gautrin, Dept of Chest Medicine, Sacré-Coeur Hospital, 5400 Gouin Blvd West, Montreal, Canada H4J 1C5.

Fax: 15143383123

\section{Keywords: Asthma}

immunological sensitization

occupational asthma

Received: July 242000

Accepted after revision December 12 2000

This study was supported by the Medical Research Council of Canada (grant no MT-12256) and the Fonds de la Recherche en Sante du Quebec.
Laboratory animals are a common cause of occupational asthma (OA) due to high-molecular-weight allergens [1]. Rat urine is the major and most potent source of inhaled antigens [1]. Although several crosssectional studies have been carried out as summarized elsewhere [1], very few longitudinal studies have followed laboratory animal workers prospectively. Cullinan et al. [2] followed a cohort of 342 previously exposed laboratory animal workers on a 6-month basis over a 3.5-yr interval. They reported incidence rates of 7.3/100 person-yrs for eye/nose symptoms, 4.1/100 person-yrs for the development of skin reactivity to rat urinary allergens, 4.8/100 person-yrs for skin symptoms and 3.5/100 person-yrs for respiratory symptoms. A cohort of 769 apprentices starting exposure to high-molecular-weight antigens, including 417 in animal-health technology programmes has previously been described [3]. In this specific programme, it was shown that 85 of the 394 participants $921.5 \%$ of persons at risk developed skin reactivity to laboratory-animal-derived allergens over the follow-up period, corresponding to 7.9/100 person-yrs [4]. It was also found that the incidence of OA defined by the presence of skin reactivity to a programme-specific antigen and significant changes in bronchial responsiveness to methacholine was $2.7 \%$ (28/1043 person-yr) [5]. The purpose of this analysis, carried out in the 394 participants in the prospective study of laboratoryanimal workers, was to describe the time-course of incident cases of skin reactivity, work-related rhinoconjunctivitis (RC) and respiratory symptoms and OA over the 3-4-yr-duration of the programme. The hypothesis was that the incidence would be higher in the period immediately after starting exposure and diminish thereafter. The value of skin reactivity and work-related $\mathrm{RC}$ was also assessed as an early marker for the development of OA. Such an approach has been advocated in the prevention of OA [6] but has not been validated.

\section{Subjects and methods}

The subjects were 417 apprentices in animal-health technology, part of a cohort of 769 apprentices (including 230 apprentices in pastry-making and 122 in dental hygiene technology) recruited from 14 specialized schools (five in animal-health technology) upon starting their training between 1993 and 1995. 
Baseline characteristics of the cohort members have been reported elsewhere [3]. Subjects in these schools were eligible for the cohort study provided that they had not been exposed to the relevant work-related aero-allergens in an apprenticeship or at work for at least three months before entering the career programme, as assessed during a preliminary visit. The duration of the animal-health technology programme was either 32 or 44 months. The majority of students $(n=307)$ entered the 32-month duration programme while the rest $(n=110)$ attended the longest programme, of 4-yrs duration. After the baseline assessment, the students were reassessed at 8, 20, 32 and 44 months after starting the programme. The majority of students attended the 20-month $(\mathrm{n}=345$, participation rate $=82.7 \%)$ and 32-month $(n=355$, participation rate $=85.1 \%$ ) visits. There were 136 students at the 8 -month assessment because the others had not been exposed to laboratory animals since starting in the programme. Finally, $98 / 110(89.0 \%)$ subjects in the 44-month duration programme attended the last visit.

The study was approved by the ethics committee of Sacré-Coeur Hospital. The subjects gave written consent to their participation when recruited in this cohort study.

At the time of entry into the apprenticeship programme and at each follow-up visit, each student answered a respiratory questionnaire derived from the standardized questionnaire of the International Union against Tuberculosis and Lung Diseases (IUATLD) [7]. At each visit, nasal, ocular, respiratory, and skin symptoms were assessed during exposure to the specific agents present during work in the laboratory, allowing the work-relatedness of the symptomatology to be assessed.

Skin-tests were performed using the skin-prick method, using 11 common inhalants: mixed trees, mixed grass, and ragweed pollens; Alternaria, Aspergillus, Hormodendrum; feathers; Dermatophagodes farinae and D. pteronyssinus; and cat and dog dander (Omega, Montreal, Canada). Histamine phosphate (1/ $200 \mathrm{~g} \cdot \mathrm{mL}^{-1}$ ) was used as a positive control, and diluent (glycerine, 50\%), as a negative control. The largest weal diameter was assessed 10-15 min after introducing the antigen. A positive reaction was defined as a weal $\geqslant 3 \mathrm{~mm}$ in the absence of reaction to the diluent and in the presence of a positive reaction to histamine phosphate.

In addition, skin-prick-tests were performed with extracts from aero-allergens potentially present in the working areas of students and confirmed as specific to the programme by the authors [4]. These consisted of urinary proteins from rat, mouse and rabbit (Pharmacia Allergon AB, Angelholm, Sweden), as well as rabbit danders (Omega). The criterion for a positive reaction to a specific allergen was the same as for common allergens. Subjects who demonstrated sensitization to a work-related antigen at the initial visit were also followed to detect possible new sensitization to another occupational allergen.

All extracts were obtained before the beginning of the study in sufficient amounts to perform the estimated number of skin-tests to the end of the project. Each extract was prepared from the same batch. The same nurse performed the skin-prick-testing throughout the study.

Spirometry with the assessment of forced expiratory volume in one second (FEV1) and forced vital capacity (FVC) was carried out using a Collins apparatus (Survey/1 Plus; Collins, Braintree, MA, USA) according to published standards [8]. Methacholine inhalation tests were performed using a Wright's nebulizer (Roxon Meditech Ltd, Montreal, Quebec, Canada) (output $=0.14 \mathrm{~mL} \cdot \mathrm{min}^{-1}$ ) at tidal volume breathing for 2 min according to guidelines slightly modified from those of the European Respiratory Society [9]. The procedure for performing the methacholine test was modified as described elsewhere to take into account the absence of an on-site physician [10]. The provocative concentration causing a 20\% fall in FEV1 (PC20) was interpolated from individual dose-response curves drawn on a semilogarithmic scale using noncumulative doses.

Reference values for FEV1 and FEV1/FVC were taken from KNUDSON et al. [11]. Bronchial hyperresponsiveness (BHR) was set at $\leqslant 16 \mathrm{mg} \cdot \mathrm{mL}^{-1}[12]$.

New sensitization was defined as changes in skin reactivity (from absent to present) occurring at one of the follow-up visits and remaining positive thereafter. New work-related symptomatology was defined in the same way. Incident cases of occupational RC were defined as subjects who developed a specific immunological sensitization to at least one work-related allergen and nasal and/or conjunctival symptoms after the baseline visit. Incident cases of OA were defined as subjects who developed a specific immunological sensitization to at least one work-related allergen and a 3.2-fold decrease in PC20 methacholine after the baseline visit [13].

\section{Statistical analysis}

The proportion of incident cases of skin reactivity, work-related symptoms, occupational RC and asthma relative to the number of subjects present at each visit was computed. The positive predictive values of skin reactivity at baseline or follow-up for subsequent and/ or concomitant outcome were calculated. This estimate was obtained by summing up the new cases of symptoms occurring at subsequent visits and half of those detected at the same visit relative to the occurrence of skin reactivity. The assumption was made that when skin reactivity and work-related symptoms were detected concomitantly, skin reactivity preceded the development of symptoms in $50 \%$ of cases. The same rationale holds for occupational $\mathrm{RC}$ in relation to OA. Statistical analyses were performed using the Statistical package for the Social Sciences (SPSS) software (SPSS version 9.1 for Windows; SPSS, Chicago, IL, USA).

\section{Results}

Table 1 shows the number of subjects with the outcomes that were examined and the percentages relative to those seen at each visit. At most visits, the incidence figures were greater for work-related $\mathrm{RC}$ 
Table 1. - Time-course of work-related symptoms and occupational diseases in apprentices exposed to laboratory animals

\begin{tabular}{|c|c|c|c|c|c|}
\hline & \multicolumn{4}{|c|}{ Years in the programme } & \multirow[t]{2}{*}{ Incidence-density* } \\
\hline & 1 & 2 & 3 & 4 & \\
\hline Number present at visit & 136 & 345 & 355 & 98 & \\
\hline \multicolumn{6}{|l|}{ Skin reactivity } \\
\hline Programme-related antigen & $14(10.3)$ & $37(10.7)$ & $29(8.2)$ & $5(5.1)$ & $85 / 1075$ (7.9) \\
\hline Mites & $4(2.9)$ & $14(4.1)$ & $14(3.9)$ & $1(1.0)$ & $33 / 716(4.6)$ \\
\hline Pets & $9(6.6)$ & $7(2.0)$ & $14(3.9)$ & $5(5.1)$ & $35 / 926(3.8)$ \\
\hline Pollens & $0(0)$ & $8(2.3)$ & $8(2.3)$ & $1(1.0)$ & $17 / 788(2.2)$ \\
\hline \multicolumn{6}{|l|}{ Work-related symptoms } \\
\hline Skin & $4(2.9)$ & $33(9.6)$ & $39(11.0)$ & $5(5.1)$ & $81 / 1051(7.7)$ \\
\hline Rhinoconjunctivitis & $17(12.5)$ & $48(13.9)$ & $30(8.5)$ & $4(4.1)$ & 99/961 (10.3) \\
\hline Respiratory & $1(0.7)$ & $11(3.2)$ & $9(2.5)$ & $1(1.0)$ & $22 / 1115(2.0)$ \\
\hline \multicolumn{6}{|l|}{ Occupational } \\
\hline Rhinoconjunctivitis & $9(6.6)$ & $24(7.0)$ & $29(8.2)$ & $0(0)$ & $62 / 1096(5.7)$ \\
\hline Asthma & $5(3.7)$ & $9(2.6)$ & $13(3.7)$ & $1(1.0)$ & $28 / 1043(2.7)$ \\
\hline
\end{tabular}

Data are presented as $\mathrm{n}(\%)$ or incidence $(\%)$. Total number of participants $=417$ except for the assessment of occupational asthma $(n=373)$ because 44 students refused the methacholine inhalation test. *: number of cases/person-yrs in the programme.

symptoms followed in order by skin reactivity to programme-related antigen, work-related skin symptoms, occupational $\mathrm{RC}$ and, almost equally, $\mathrm{OA}$ and work-related respiratory symptoms. The incidence of programme-related antigen skin reactivity and RC symptoms was higher at 1-2 yrs after entering the programme, while it was higher at $2-3$ yrs for respiratory symptoms and at 3 yrs for occupational $\mathrm{RC}$. The percentages of new events were lower for all outcomes in the fourth yr after entering the programme.

The percentage of new cases of OA were comparable in years 1-3 of the programme. The percentage of incident cases of sensitization to a programme-related antigen in the fourth yr of the programme $(5.1 \%)$ was comparable to the incidence of sensitization to mites $(4.6 \%)$ and pets $(3.8 \%)$ during the study period. Onehundred and twenty-nine subjects had skin reactivity to a programme-related antigen at the end of follow-up, including 44 at baseline and 85 incident cases [4]. Of the 85 incident cases of sensitization to an animal-derived antigen, 79 (93\%) had methacholine inhalation testing at one or more follow-up visits. Of the 203 subjects who underwent methacholine testing at one or more followup visits, $53(26.1 \%)$ dropped their $\mathrm{PC} 20$ values by at least a 3.2-fold difference. Of these 53, $28(43 \%)$ were cases of OA and represent the burden of occupational exposure on all incident cases of asthma in this cohort. Four of the remaining 25 individuals were incident cases of sensitization to mites $(\mathrm{n}=2)$ or pets [3] (none to pollens or moulds). Therefore, 28 of the 373 subjects $(7.5 \%)$ in the cohort satisfied the definition of OA.

Table 2 gives the number of incident cases of workrelated symptoms and the timing of the occurrence of symptoms in relation to skin reactivity. The positive predictive values (PPVs) were higher for RC symptoms than for respiratory symptoms. It is estimated that 27 (i.e. $17+(19 / 2)$ in the nasal and/or ocular symptom line

Table 2. - Incidence of work-related symptoms in relation to the time of occurrence of skin reactivity to laboratory animalderived antigens

\begin{tabular}{|c|c|c|c|c|c|c|}
\hline & \multicolumn{5}{|c|}{ Skin reactivity } & \multirow[t]{3}{*}{$\mathrm{PPV}^{*}$} \\
\hline & \multicolumn{4}{|c|}{ Present } & \multirow[t]{2}{*}{ Absent } & \\
\hline & $\begin{array}{l}\text { Before } \\
\text { symptoms }\end{array}$ & $\begin{array}{l}\text { Same time } \\
\text { as symptoms }\end{array}$ & $\begin{array}{c}\text { After } \\
\text { symptoms }\end{array}$ & $\begin{array}{c}\text { No } \\
\text { symptoms }\end{array}$ & & \\
\hline Cutaneous symptoms & 21 & 22 & 7 & 67 & 31 & $32 / 11728(19-35)$ \\
\hline \multicolumn{7}{|l|}{ Work-related RC symptoms } \\
\hline Nasal & 18 & 17 & 13 & 42 & 44 & $27 / 9030(21-41)$ \\
\hline Ocular & 14 & 16 & 17 & 59 & 31 & $22 / 10621(14-30)$ \\
\hline Nasal and/or ocular & 17 & 19 & 17 & 26 & 46 & $27 / 9030(21-41)$ \\
\hline \multicolumn{7}{|c|}{ Work-related respiratory symptoms } \\
\hline Dyspnoea & 7 & 1 & 5 & 110 & 8 & $8 / 1236.5(3-13)$ \\
\hline Wheezing & 8 & 4 & 6 & 111 & 3 & $10 / 1298.0(4-14)$ \\
\hline Dyspnoea and/or wheezing & 9 & 4 & 6 & 103 & 3 & $11 / 229.0(5-16)$ \\
\hline
\end{tabular}

Data are presented as n except for positive predictive values (PPV) which show incidence calculated as described below and \% $(95 \%$ confidence interval (CI)). *: The numerator is the total of (all incident cases of symptoms detected after the occurrence of skin reactivity) + (half of incident cases of symptoms detected at the same time as skin reactivity) (see text for rationale); the denominator is the number of subjects with skin reactivity at baseline or at follow-up and without the pertinent symptom at baseline. CI values are computed under the naive assumption that PPV is a simple proportion. 
of table 2) of the 99 incident cases of work-related RC showed skin reactivity to a programme-related antigen before symptoms. Eleven (i.e. $9+(4 / 2)$ in the dyspnoea and/or wheezing line of table 2), of the $22(50 \%)$ incident cases of work-related respiratory symptoms had skin reactivity to a programme-related antigen before the onset of symptoms. Nineteen of the 122 cases $(15.6 \%)$ with skin reactivity who were at risk developed respiratory symptoms during the course of the study. The PPV of work-related RC symptoms for the development of OA was $11.4 \%$ while the PPV of work-related respiratory symptoms for the development of OA was $25 \%$.

\section{Discussion}

In this study it was found that the peak incidences of skin reactivity and work-related RC symptoms are maximal 1-2 yrs after entering a programme, while occupational RC defined by the occurrence of both skin reactivity and work-related $\mathrm{RC}$ is at its peak 3 yrs after entering the programme. The number of cases of $\mathrm{OA}$ is comparable throughout the first $3 \mathrm{yrs}$.

There are few longitudinal studies of workers exposed to laboratory antigens and none in which subjects were followed from the beginning of exposure and assessed for symptoms, immunological reactivity and bronchial responsiveness. Results on the incidence and risk factors for immunological sensitization were recently reported for a cohort of 769 apprentices [4]. Cullinan et al. [2] prospectively assessed 342 laboratory animal workers. In contrast to the present study subjects, these workers had up to 4 yrs of exposure to laboratory animals before the onset of the study and bronchial responsiveness was not assessed. The incidence-density in the present study for programmerelated antigen skin reactivity (7.9 per 100 person-yrs) was twice that found by Cullinan et al. [2] (4.1 per 100 person-yrs). The incident-density of RC symptoms was also higher in the presented study: 12.0 per 100 person-yrs as compared with 7.3 per 100 person-yrs in the study of Cullinan et al. [2]. It was found that the percentage of new cases of skin reactivity and $\mathrm{RC}$ symptoms were higher in the first 2 yrs of the programme; Cullinan et al. [2] reported similar findings in their cohort of workers after they started new employment. The higher incidence figures in the present study compared with those from the study by Cullinan et al. [2] could be explained by the fact that in the present study, the subjects were assessed earlier after starting exposure for the development of outcomes than in the British study, thus minimizing a potential survival bias. Furthermore, the apprentices studied here were exposed to three species of rodents, and therefore the potential for developing new workrelated antigen skin reactivity was increased; indeed, if only incident skin reactivity to rat was considered, the incidence density would be 5.9 per 100 person-yrs. It is interesting to note that the incidence of sensitization in the fourth year of the programme $(5.1 \%)$ is of the same order of magnitude as the incidence density of sensitization to common allergens $(4.6 \%$ for mites and $3.8 \%$ for pets) throughout the study. Therefore, after a rise in the first 2 yrs in the programme, the incidence of sensitization decreases in the third yr and reaches the incidence of skin reactivity to common allergens after 4 yrs. This pattern has relevant significance in term of prevention. Indeed, if the decision to screen for sensitization is made, the relevant timing should be in the first 3 yrs of exposure. Theoretically, it is logical to assume that immunological reactivity to a work-related antigen would develop first, followed by the involvement of the target organ, i.e. the nose/eye and respiratory tract. From the present data, it appears that this represents the time-course model in the case of occupational RC, whose highest incidence occurred later than the highest incidence of work-related antigen skin reactivity. The theoretical chronological pattern was not found in the case of OA; this is probably difficult to interpret because there was not a sufficient number of cases. Also, shorter time interval between assessments of bronchial responsiveness may be indicated. The low PPVs of skin reactivity for the development of symptoms and diseases suggest that the theoretical model of the natural history of OA is not satisfactory. This suggests that chronological events may follow another pattern e.g. symptoms may precede the onset of skin reactivity. However, it must be mentioned that the method used to calculate the predictive value may have resulted in an underestimation. Indeed, it is hypothesized that skin reactivity preceded the development of symptoms in $50 \%$ of cases when skin reactivity and work-related symptoms were detected concomitantly.

It is unlikely that the described time-course of sensitization, symptoms and disease had been affected by an attrition bias. Only 36/417 subjects $(8.6 \%)$ left the programme and work-related symptoms during training were not associated with quitting [14]. Only two subjects, the two in the animal-health technology programme, reported leaving due to work-related RC symptoms. The proportion of apprentices who reported at least one of the skin, $\mathrm{RC}$ or chest symptoms was not different in quitters $(7 / 27,26 \%)$ and in those who stayed $(94 / 370,25 \%)$. It is also unlikely that the pattern of sensitization and symptoms could have been affected by an exposure pattern with enhanced exposure in the first $2 \mathrm{yrs}$ of the programme. The students were indeed exposed to rodents, the main sensitizer [4] for, on average, $14 \mathrm{~h}$ in the first yr, $10 \mathrm{~h}$ in the second and $91 \mathrm{~h}$ in the third. There were also no changes in animal housing facilities during the course of the programme. Finally, PPVs were estimated. Since this study is a cohort the PPV is not biased by artificial prevalence as in case-control studies.

Secondary prevention of OA is based on the identification of cases at an early stage. For this, two tools have been considered. First, as immunological reactivity theoretically precedes the onset of $\mathrm{OA}$ with a latency period, assessment of skin reactivity was proposed [15]. Second, RC symptoms generally precede the onset of OA [16] and can, therefore, be suggested in a prevention strategy. Specific sensitization is highly associated with the development of work-related symptoms (statistically significant relative risks $(95 \%$ confidence intervals) of $6.8(4.2-11.1)$ for RC and $4.9(2.2-10.8)$ for respiratory symptoms (unpublished 
data)). Although it was found that skin reactivity had a low predictive value for work-related RC (30\%) and respiratory symptoms $(9 \%)$, the data also show that $50 \%$ of the 22 incident cases of work-related respiratory symptoms had skin reactivity to a programme-related antigen before the development of symptoms. Also, 22 of the 122 cases $(18.0 \%)$ with skin reactivity who were at risk developed respiratory symptoms during the course of the study. Moreover, the predictive value of RC symptoms in regards to the development of respiratory symptoms was $30 \%$, which is not negligible. Finally, a follow-up of 4 yrs is relatively short as incident cases of work-related symptoms may occur later. This being said, taken together, these data suggest that assessment of skin reactivity and RC symptoms should still be considered in the context of screening programmes. The use of skin reactivity as a predictor for symptoms and diseases and the use of RC symptoms as a predictor of the development of lower respiratory symptoms also needs to be assessed from the points of view of cost-effectiveness and compliance.

In conclusion, this description of the pattern of the natural history of sensitization, symptoms and diseases in apprentices starting exposure to laboratory animals, should be examined in more depth to confirm the temporal trends.

Acknowledgements. The authors express their gratitude to the technicians, principally $\mathrm{J}$. L'Archeveque, and to nurse M. Magnan, who played a key role in the recruitment of subjects and the handling of results. They also thank all those responsible at the participating teaching institutions and all of the students who took part in the study. Finally, they acknowledge the collaboration of L. Schubert for reviewing the manuscript.

\section{References}

1. Gordon S, Newman Taylor AJ. Animal, insect, and shellfish allergy. In: Bernstein IL, Chan-Yeung M, Malo J-L, Bernstein DI, eds. Asthma in the workplace. New York, Marcel Dekker Inc., 1999; pp. 399-424.

2. Cullinan $\mathrm{P}$, Cook A, Gordon $\mathrm{S}$, et al. Allergen exposure, atopy and smoking as determinants of allergy to rats in a cohort of laboratory employees. Eur Respir J 1999; 13: 1139-1143.

3. Gautrin D, Infante-Rivard C, Dao TV, MagnanLarose M, Desjardins D, Malo J-L. Specific IgEdependent sensitization, atopy and bronchial hyperresponsiveness in apprentices starting exposure to protein-derived agents. Am J Respir Crit Care Med 1997; 155: 1841-1847.
4. Gautrin D, Ghezo H, Infante-Rivard C, Malo $\mathrm{J}$-L. Incidence and determinants of IgE-mediated sensitization in apprentices: a prospective study. $\mathrm{Am}$ J Respir Crit Care Med 2000; 162: 1222-1228.

5. Gautrin D, Infante-Rivard C, Ghezzo H, Malo $\mathrm{J}$-L. Incidence and host determinants of probable occupational asthma in apprentices exposed to laboratory animals. Am $\mathrm{J}$ Respir Crit Care Med 2001; 163: 899-904.

6. Malo J-L, Blanc P. Occupational lung diseases: issues in management. Eur Respir Mon 1999; 11: 106-123.

7. Burney PGJ, Laitinen LA, Perdrizet S, et al. Validity and repeatability of the IUATLD (1984) bronchial symptoms questionnaire: an international comparison. Eur Respir J 1989; 2: 940-945.

8. American Thoracic Society. Standardization of spirometry. Am J Respir Crit Care Med 1995; 152: 1107-1136.

9. Sterk PJ, Fabbri LM, Quanjer PH, et al. Airway responsiveness. Standardized challenge testing with pharmacological, physical and sensitizing stimuli in adults. Report working party standardization of lung function tests European Community for Steel and Coal. Official statement of the European Respiratory Society. Eur Respir J 1993; 6: Suppl. 16, 53-83.

10. Troyanov S, Malo J-L, Cartier A, Gautrin D. Frequency and determinants of exaggerated bronchoconstriction due to methacholine inhalation in epidemiological and clinical setups. Eur Respir J 2000; 16: 9-14.

11. Knudson RJ, Lebowitz MD, Holberg CJ, Burrows B. Changes in the normal maximal expiratory flowvolume curve with growth and aging. Am Rev Respir Dis 1983; 127: 725-734.

12. Malo J-L, Pineau L, Cartier A, Martin RR. Reference values of the provocative concentrations of methacholine that cause $6 \%$ and $20 \%$ changes in forced respiratory volume in one second in a normal population. Am Rev Respir Dis 1983; 128: 8-11.

13. Dehaut P, Rachiele A, Martin RR, Malo J-L. Histamine dose-response curves in asthma: reproducibility and sensitivity of different indices to assess response. Thorax 1983; 38: 516-522.

14. Monso E, Malo J-L, Infante-Rivard C, et al. Individual characteristics and quitting in apprentices exposed to high-molecular-weight agents. Am J Respir Crit Care Med 2000; 161: 1508-1512.

15. Malo J-L. Occupational asthma. In: Hirsch A, Goldberg M, Martin JP, Masse M, eds. Prevention of respiratory diseases. Lung biology in health and disease series. Vol 68. New York, Marcel Dekker Inc., 1993; pp. 117-131.

16. Malo J-L, Lemière $\mathrm{C}$, Desjardins $\mathrm{A}$, Cartier A. Prevalence and intensity of rhinoconjunctivitis in subjects with occupational asthma. Eur Respir $J$ 1997; 10: 1513-1515. 